\title{
From atomic clocks to coordinate times
}

\author{
Gérard Petit \\ Bureau Intenational des Poids et des Mesures, \\ Pavillon de Breteuil, F-92312 Sèvres Cedex, France \\ email: gpetit@bipm.org
}

\begin{abstract}
With the present level of accuracy it may be necessary to use space borne atomic clocks to directly generate TCG, and to start addressing the limitations in the coordinate time transformations.
\end{abstract}

Keywords. time, relativity, standards

The IAU1991 Resolution A4, complemented by IAU2000 Resolution B1.3-4, provide rigorous definitions for barycentric and geocentric reference systems in a relativistic framework and define the coordinate times of these systems as TCB and TCG, respectively. Other coordinate times in use are TT, defined from TCG through IAU2000 Resolution B1.9, and TDB, whose rigorous definition from TCB has been adopted in 2006.

For practical use, these coordinate times must be realized and the proper time provided by atomic clocks (Atomic time AT) generates all coordinate times. The present sequence is $\mathrm{AT}=>\mathrm{TT} \equiv>\mathrm{TCG} \rightarrow \mathrm{TCB} \equiv>\mathrm{TDB}$, where $\equiv>$ is an exact transformation and where $=>$ indicates the complex series of operations involved in generating International atomic time TAI.

The present uncertainty of realization of TAI and of TT(BIPM), another realization of $\mathrm{TT}$, is about $1 \times 10^{-15}$ in frequency with good prospects to reach $1 \times 10^{-16}$ in the coming years. The uncertainty brought by the transformation TCG $\rightarrow$ TCB is below $1 \times 10^{-17}$. Future evolutions of atomic clocks, e.g., using new optical transitions, could allow reaching this level and will bring new performances to the generation of coordinate time scales.

At this level of accuracy it may be necessary to use space borne atomic clocks to directly generate TCG, and to start addressing the limitations in the coordinate time transformations. 